\title{
A REVEALED-PREFERENCE IMPLICATION OF WEIGHTED UTILITY DECISIONS UNDER UNCERTAINTY \\ by
}

In-Uck Park

Discussion Paper No. 270, September 1993

Center for Economic Research

Department of Economics University of Minnesota Minneapolis, MN 55455 


\title{
A Revealed-Preference Implication of Weighted Utility Decisions under Uncertainty
}

\author{
In-Uck Park* \\ Department of Economics \\ University of Minnesota \\ 271 19th Avenue S. \\ Minneapolis, MN 55455 \\ U.S.A. \\ and \\ Department of Economics \\ University of Bristol \\ 8 Woodland Road \\ Bristol, BS8 1TN \\ U.K.
}

September, 1993

\begin{abstract}
Revealed preference of the weighted utility theory of Chew (1983) is investigated in the same set-up as Green and Osband (1991) for expected utility theory; the structure of the partition of the state simplex according to the chosen act is examined. It is shown that the boundary between two partition elements generated by a weighted utility is the solution set to a quadratic equation. Moreover, except for special "symmetric" pairs of acts, weighted utilities in a generic set produce revealed preference partitions with nonaffine boundaries, so that they are distinguishable from those of expected utilities which have affine boundaries according to Green and Osband.

Keywords: revealed preference, expected utility, weighted utility, behavioral partition, border of acts

$J E L$ classification code: D81

Correspondence: University of Bristol address above
\end{abstract}

- I thank Ed Green for the motivation and continued encouragement as well as helpful comments and Andy McLennan for beneficial discussions. I am responsible for remaining errors. 


\section{Introduction}

The expected utility theory which dates back to Bernoulli and revived by von Neumann and Morgenstern (1944) and by Savage (1954), has been the standard analytic framework of decision under uncertainty. However, some researchers have suggested that people violate expected-utility maximization in systematic ways, for instance, Allais' paradox. Such reservations about expected utility theory have led various authors to develop alternative theories that generalize the expected utility theory. ${ }^{(1)}$ These are called "nonexpected utility theories."

Usually, individuals' preferences are not observable directly. But, they can be inferred from people's behavior if certain preferences generate distinctive behavior pattern. So, the expected utility theory and its alternative theories can be evaluated systematically as descriptions of behavior, using a revealed preference approach that is well established in the study of choice under certainty. This paper investigates the revealed preference of the weighted utility theory of Chew (1983), the closest to the expected utility among alternative theories, within a certain set-up specified below and compares with the corresponding characterization of expected utility theory by Green and Osband (1991). The main result is that these two theories induce systematically different characterizations, and the revealed preference of weighted utilities is distinguishable from that of expected utilities in a "generic" sense unless the observed acts are "symmetric" in the sense specified later.

The expected utility theory basically says that the preference over random prospects is representable by the expected value of the utility levels of the pure consequences which may be realized according to the random prospects; the preference function is "linear" in the probabilities of pure consequences. This linearity of preference is in essence a consequence of the so-called "Independence axiom." Chew introduces weighted utility theory by basically replacing the Independence axiom with a weaker concept called "Betweenness axiom." It generalizes the expected utility theory by including preferences over random prospects which are representable by the mean of the utility levels of pure consequences where the weights are parametrized by an additional "weighting function."

(1) Chew (1983), Dekel (1986), Fishburn (1983) and Quiggin (1982) among others. 
The decision making set-up in this paper follows Green and Osband (1991) in their characterization of revealed preference of expected utility: A decision maker has a finite set of acts which induce state-contingent consequences. Facing uncertainty represented by a probability measure over states, the decision maker chooses a best act in the sense that the consequence (a probability measure over pure consequences) that this act induces is preferred to the ones induced by other acts according to his or her preference over random prospects. Then, the unit simplex of states is partitioned according to the chosen act. This partition is called a behavioral partition. The subject of the study is a structural characterization of behavioral partitions when the preferences admit a weighted utility representation. ${ }^{(2)}$

Green and Osband completely characterize the behavioral partition induced by a preference conforming to the expected utility theory roughly as "consisting of convex polyhedral partition elements satisfying a certain integrability condition." In particular, the boundary between two partition elements (this boundary represents the set of probability assessments over states for which the acts chosen in either elements are indifferent) is a subset of an affine surface.

A preference is defined over random prospects which are represented by points in the unit simplex of consequences. The structure of a behavioral partition is determined by the underlying preference. A preference that admits an expected utility representation ("an expected utility," hereafter) is characterized as generating indifference surfaces in the unit simplex of consequences which are affine and parallel to each other, while a preference that admits a weighted utility representation ("a weighted utility," hereafter) generates indifference surfaces which are affine with a common axis. (See Figure 1.) Even though the Betweenness axiom guarantees affine indifference surfaces for both preferences, this axiom alone does not produce a behavioral partition with affine boundaries; it is shown that a boundary between partition elements produced by a weighted utility is a quadratic surface.

(2) Another decision making situation explored in the study of the revealed preference of the expected utility theory is to observe the choice of acts by changing the set of feasible acts while fixing the probability distribution of the states. This is used in Border (1992), Fishburn (1975), Kim (1991) and Ledyard (1986) among others. 
Since affine surfaces are a special form of quadratic surfaces, a weighted utility may produce a behavioral partition that is not distinguishable from those produced by expected utilities. An example in section 2 illustrates this possibility. However, it is also shown that this phenomenon is only exceptional; the boundary of partition elements produced by a weighted utility is non-linear except for a small subset of weighted utilities whose closure has measure zero in a properly defined space of weighted utilities. Therefore, the revealed preference of a generic weighted utility is distinguishable from those of expected utilities.

An example may illustrate a behavior which is consistent with the weighted utility theory but not with the expected utility theory. Consider the following behavior of a Londoner who decides whether to take an umbrella with him after listening (and believing) the weather forecast; he takes an umbrella if the forecast is either "rain for sure" or "sunny for sure," but decides not to take one if "even chance of rain and sunny." While this behavior is impossible under an expected utility, it is normal according to the weighted utility theory, that is, there is a nonempty open subset of weighted utilities from which this behavior results.

The rest of the paper is organized as follows. Section 2 formulates the model and reviews the revealed preference of expected utilities discovered by Green and Osband. Section 3 establishes a necessary condition for a behavioral partition to be consistent with a weighted utility. Section 4 identifies special pairs of acts such that the choice behavior between these two acts produced by weighted utilities is not distinguished from that produced by expected utilities. Section 5 shows the generic distinction of the two theories in revealed preference provided that the observed acts are not "special" as identified in section 4. Section 6 contains some concluding remarks.

\section{Model, Preferences and Revealed Preference of Expected Utility}

Let $\mathbf{S}=\left\{s_{1}, \ldots, s_{n}\right\}$ be a set of states of nature and $\mathbf{A}$ be a finite set of acts. The simplex of $\mathbf{S}, \Delta(\mathbf{S})=\left\{p \in \mathbb{R}^{n}: p_{j} \geq 0\right.$ and $\left.\sum_{j=1}^{n} p_{j}=1\right\}$, is the space of probability assessments that an agent may have regarding the state of nature. A behavior pattern is a function $\beta: \Delta(\mathbf{S}) \rightarrow \mathbf{A}$. The collection of inverse images of $\beta$ is a partition of $\Delta(\mathbf{S})$. This collection is called the behavioral partition of $\Delta(\mathbf{S})$ by $\beta$, and each element of the partition 
is called a cell.

For each state, each act leads to a pure consequence. Hence, an act is a function from $S$ to the set of consequences $Z$. A decision maker's preference $\succeq$ is defined on the random prospects over $\mathbf{Z}$, that is, $\succeq \subset \Delta(\mathbf{Z}) \times \Delta(\mathbf{Z})$. For $\sigma, \sigma^{\prime} \in \Delta(\mathbf{Z})$, we write $\sigma \succeq \sigma^{\prime}$ to mean $\left(\sigma, \sigma^{\prime}\right) \in \succeq$. Given a preference $\succeq$, the set of best acts for $p \in \Delta(\mathbf{S})$ is $B R(p ; \succeq)=\{a \in \mathbf{A}: a(p) \succeq b(p)$ for all $b \in \mathbf{A}\}$. Faced with an assessment $p \in \Delta(\mathbf{S})$, a decision maker chooses an act in $B R(p ; \succeq)$. A preference $\succeq$ rationalizes a behavior pattern $\beta: \Delta(\mathbf{S}) \rightarrow \mathbf{A}$ if

i) $\beta(p) \in B R(p ; \succeq)$ for all $p \in \Delta(\mathbf{S})$, and

ii) for all $a \in \mathbf{A}$, there exists $p \in \Delta(\mathbf{S})$ such that $B R(p ; \succeq)=\{a\} .^{(3)}$

A preference $\succeq$ rationalizes a partition of $\Delta(\mathbf{S})$ if it is the behavioral partition of a behavior pattern rationalized by $\succeq$.

However, an outsider generally does not know which act leads to which consequence. Given $\mathbf{S}, \mathbf{A}$ and a set of consequences $\mathbf{Z}$, consequence assignment on $\mathbf{Z}$ refers to assigning for each pair $(s, a) \in \mathbf{S} \times \mathbf{A}$, a consequence in $\mathbf{Z}$, so that each act is a function from $\mathbf{S}$ to $\mathbf{Z}$.

Definition 2.1 : Let $\mathrm{S}$ be a finite set of states of nature and $\mathrm{A}$ be a finite set of acts. $A$ partition II of $\Delta(\mathbf{S})$ is $E U$-rational (WU-rational, respectively) if there exist a set of consequences $\mathbf{Z}$, a consequence assignment on $\mathbf{Z}$ and a preference $\succeq$ on $\Delta(Z)$ conforming to the expected utility theory (weighted utility therory, respectively) that rationalize II.

Green and Osband (1991) completely characterizes the structure of EU-rational behavioral partitions. The main question in this paper is a comparison of behavioral partitions between weighted utility and expected utility. These two theories are reviewed below for preferences defined on random prospects over a finite set $\mathbf{Z}$ of consequences.

Expected utility representation : $A$ preference $\succeq$ on $\Delta(Z)$ is represented by an expected utility if there exists a function $U: \mathbf{Z} \rightarrow \mathbb{R}$ (an expected utility function) such that

(3) Without condition $i$ ), every partition of $\Delta(\mathbf{S})$ is rationalized by the trivial preference according to which all consequences are indifferent. 
for all $\sigma, \sigma^{\prime} \in \Delta(\mathbf{Z})$,

$$
\sigma \succeq \sigma^{\prime} \Longleftrightarrow \sum_{z \in Z} U(z) \sigma(z) \geq \sum_{z \in Z} U(z) \sigma^{\prime}(z)
$$

The axiomatization of expected utility representation is well known; a preference $\succeq$ on $\Delta(Z)$ is represented by an expected utility if and only if the strict preference $\succ$ is a weak order, and the Archimedean (or continuity) axiom and the Independence (or substitution) axiom are satisfied. Chew (1983) replaced the Independence axiom with weaker axioms of Betweenness and Substitution-independence, and provided weighted utility representation. Chew's original representation is for a continuum of consequences. Here, we state for finite consequences.

Weighted utility representation : $A$ preference $\succeq$ on $\Delta(Z)$ is represented by a weighted utility if there exist functions $u: \mathbf{Z} \rightarrow \mathbb{R}$ and $\alpha: \mathbf{Z} \rightarrow \mathbb{R}_{++}$(a utility function and a weighting function) such that, for all $\sigma, \sigma^{\prime} \in \Delta(\mathbf{Z})$,

$$
\sigma \succeq \sigma^{\prime} \Longleftrightarrow \frac{\sum_{z \in Z} \alpha(z) u(z) \sigma(z)}{\sum_{z \in Z} \alpha(z) \sigma(z)} \geq \frac{\sum_{z \in Z} \alpha(z) u(z) \sigma^{\prime}(z)}{\sum_{z \in Z} \alpha(z) \sigma^{\prime}(z)}
$$

\section{Figure 1}

Expected utility

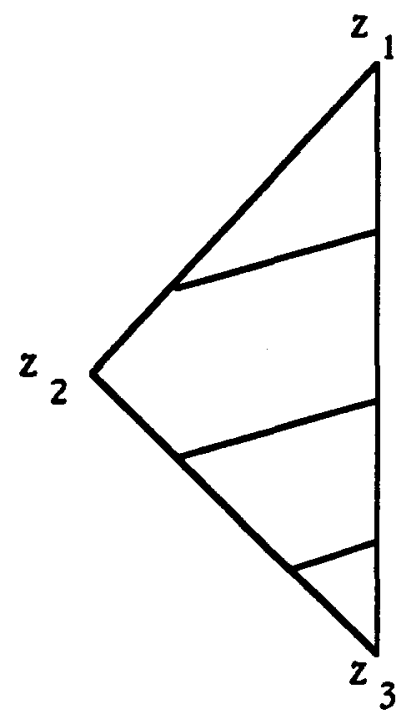

\section{Weighted utility}

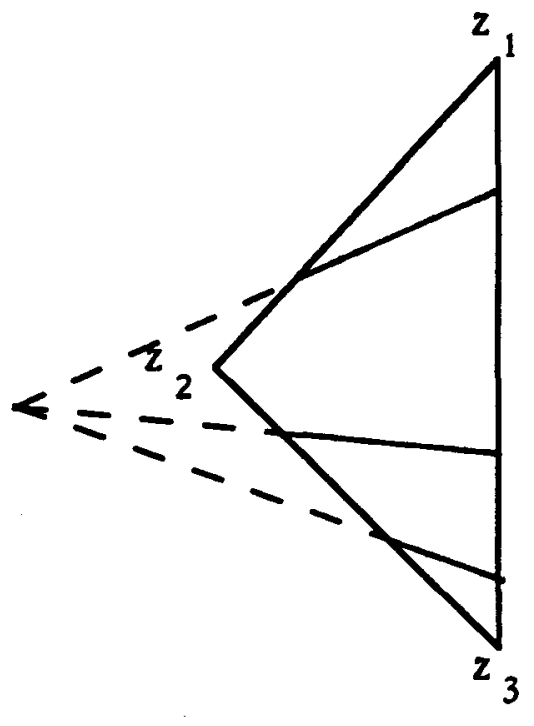


We refer precise formulation of above mentioned axioms to known references on choice theory (e.g, Kreps (1988)) and Chew (1983). Here, we discuss implication of these axioms on indifference surfaces in $\Delta(\mathbf{Z})$. Independence axiom generates indifference surfaces which are affine and parallel to one another. Betweenness implies affine indifference surfaces, and Substitution-independence implies that indifference surfaces have a "common axis" outside of $\Delta(\mathbf{Z})$ (so-called "fan-shaped" indifference surfaces). Typical indifference surfaces for both theories are presented in Figure 1 for three consequences case.

To reproduce the characterization of Green and Osband on EU-rational behavioral partition of $\Delta(\mathbf{S})$, we need some terminology. Let II be a partition of $\Delta(\mathbf{S})$. A partition element $\pi \in \Pi$ is a cell. The closure of a cell $\pi$ in $\Delta(\mathbf{S})$ is denoted by $\operatorname{cl}(\pi)$. The boundary between two cells $\pi$ and $\psi$, denoted by $B_{\pi \psi}$, is $\operatorname{cl}(\pi) \cap \operatorname{cl}(\psi)$. Two cells $\pi$ and $\psi$ are adjacent if $B_{\pi \psi}$ contains a manifold of dimension $\# \mathbf{S}-2$. A circuit is a finite sequence of successively adjacent cells such that initial and terminal cells are identical.

Proposition 2.2 (Green and Osband) : A finite partition $\Pi$ of $\Delta(\mathbf{S})$ is EU-rational if and only if the following conditions are satisfied;

(a) For each cell $\pi, \operatorname{cl}(\pi)$ is a convex polyhedral set whose relative interior in $\Delta(\mathbf{S})$ is nonempty.

(b) There are non-zero flow vectors $d(\pi, \psi)$ for all ordered pairs $(\pi, \psi)$ of adjacent cells such that $i) d(\pi, \psi)=-d(\psi, \pi)$, ii) $d(\pi, \psi)$ is orthogonal to the linear subspace of $\mathbb{R}^{\mathrm{S}}$ spanned by the parallel translation of $B_{\pi \psi}$ to be a linear subspace and the vector $(1, \cdots, 1) \in \mathbb{R}^{\mathrm{S}}$, and $\left.i i i\right)$ for any circuit of $I$, the sum of flow vectors along the circuit is 0 .

One prominent feature of EU-rational partitions is that each cell is approximated by polyhedral set so that the boundary between two adjacent cells is affine. From practical viewpoint, it is easier to check than condition (b) which is an integrability condition. In this paper, we focus on the shape of the boundary of adjacent cells of WU-rational partitions and compares with that of EU-rational partitions. Recall that both expected utility and weighted utility exhibit affine indifference surfaces. Example 2.3 shows that the naive conjecture that "affine indifference surfaces generate affine boundaries in the behavioral 
partition" is false.

Example 2.3 : Let $\mathbf{S}=\left\{s_{1}, s_{2}, s_{3}\right\}$ be states and $\mathbf{Z}=\left\{z_{1}, z_{2}, z_{3}\right\}$ be consequences. Consider two acts $a$ and $b$ :

$$
a:\left\{\begin{array}{l}
s_{1} \rightarrow z_{3} \\
s_{2} \rightarrow z_{1} \\
s_{3} \rightarrow z_{1}
\end{array} \quad b:\left\{\begin{array}{l}
s_{1} \rightarrow z_{2} \\
s_{2} \rightarrow z_{3} \\
s_{3} \rightarrow z_{1}
\end{array}\right.\right.
$$

Let $p=(0,0,1), q=(1 / 2,1 / 4,1 / 4)$ and $r=(2 / 3,1 / 3,0)$ in $\Delta(\mathbf{S})$. (See Figure $2(\mathrm{a}))$ Then,

$$
\left\{\begin{array} { l } 
{ a ( p ) = ( 1 , 0 , 0 ) } \\
{ a ( q ) = ( 1 / 2 , 0 , 1 / 2 ) } \\
{ a ( r ) = ( 1 / 3 , 0 , 2 / 3 ) }
\end{array} \quad \left\{\begin{array}{l}
b(p)=(1,0,0) \\
b(q)=(1 / 4,1 / 2,1 / 4) \\
b(r)=(0,2 / 3,1 / 3)
\end{array}\right.\right.
$$

Consider a preference $\succeq$ represented by a weighted utility such that $z_{3} \succ z_{2} \succ z_{1}$ and indifference surfaces are as shown in Figure $2(\mathrm{~b})$. Notice $b(r) \succ a(r)$ and $q$ is a convex combination of $p$ and $r$. Hence, the boundary between $a$ and $b$ in $\Delta(S)$ goes through $p$ and $q$ but not $r$. This implies that the boundary between $a$ and $b$ is not affine.

Figure 2
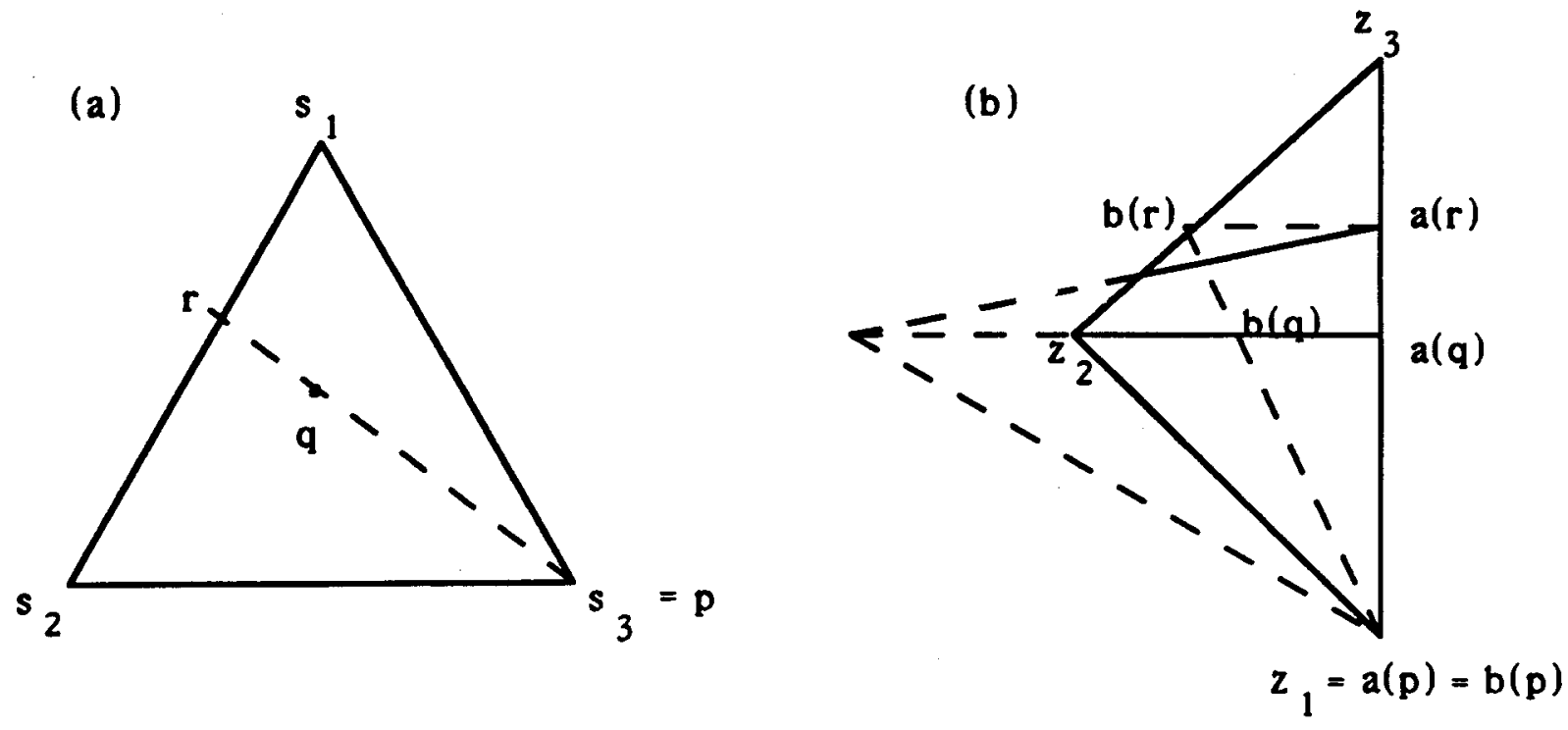


\section{A Necessary Condition for WU-rational Behavioral Partition}

Given a behavioral partition generated from a "continuous" preference, the boundary of two adjacent cells is the set of probability assessments over states of nature for which the two acts chosen in each of the adjacent cells are indifferent as best acts. Hence, we investigate the shape of the set of probability assessments over states for which two acts lead to indifferent consequences.

Let $\mathbf{S}=\left\{s_{1}, \ldots, s_{n}\right\}$ be states and $\mathbf{Z}$ be consequences. Let $\mathbf{A}$ be a finite set of acts which are functions from $\mathbf{S}$ to $\mathbf{Z}$. For $a \in \mathbf{A}$, let $a_{i}$ denote the consequence that act $a$ leads to in state $s_{i}$. We express $p \in \Delta(\mathbf{S})$ as a column vector $p=\left(p_{1}, \ldots, p_{n}\right)$ where $p_{i}$ is the probability of state $s_{i}$. For $p \in \Delta(\mathbf{S})$ and $a \in \mathbf{A}$, let $a(p) \in \Delta(\mathbf{Z})$ denote the probability distribution over consequences that $a$ generates under $p: a(p)(z)=\sum_{\left\{i: a_{i}=z\right\}} p_{i}$ for all $z \in \mathbf{Z}$. Given a preference $\succeq$ on $\Delta(\mathbf{Z})$ and acts $a$ and $b$ in $\mathbf{A}$, define the border of $a$ and $b$ as

$$
I_{a b} \equiv\{p \in \Delta(\mathbf{S}): a(p) \sim b(p)\}
$$

The shape of $I_{a b}$ when the underlying preference is a weighted utility is characterized as follows.

Proposition 3.1 : Let $\mathbf{S}=\left\{s_{1}, \ldots, s_{n}\right\}, \mathbf{A}$ and $\mathbf{Z}=\{1, \ldots, K\}$ be finite sets of states, acts and consequences, respectively. Let $\succeq$ on $\Delta(Z)$ is represented by a weighted utility. Then, for two acts $a$ and $b$ in $\mathbf{A}$,

$$
I_{a b}=\left\{p \in \Delta(\mathbf{S}): p Q p^{t}=0\right\}
$$

for some $n \times n$ symmetric matrix $Q$.

Proof : Let $u=\left(u_{1}, \ldots, u_{K}\right)$ and $\alpha=\left(\alpha_{1}, \ldots, \alpha_{K}\right)$ represent $\succeq$. The weighted utility level of $\sigma \in \Delta(\mathbf{Z})$ is

$$
\frac{\sum_{k=1}^{K} \alpha_{k} u_{k} \sigma_{k}}{\sum_{k=1}^{K} \alpha_{k} \sigma_{k}}
$$

Consider $a(p) \in \Delta(\mathbf{Z})$. Notice $\sum_{k=1}^{K} \alpha_{k} u_{k} a(p)_{k}=\sum_{i=1}^{n} \alpha_{a_{i}} u_{a_{i}} p_{i}$ and $\sum_{k=1}^{K} \alpha_{k} a(p)_{k}=$ $\sum_{i=1}^{n} \alpha_{a_{i}} p_{i}$. Hence,

$$
I_{a b}=\left\{p \in \Delta(\mathbf{S}): \frac{\sum_{i=1}^{n} \alpha_{a_{i}} u_{a_{i}} p_{i}}{\sum_{i=1}^{n} \alpha_{a_{i}} p_{i}}=\frac{\sum_{i=1}^{n} \alpha_{b_{i}} u_{b_{i}} p_{i}}{\sum_{i=1}^{n} \alpha_{b_{i}} p_{i}}\right\}
$$


After rearrangement, the equality of weighted utility levels in (3.c) is a polynomial equation in $p_{i}$ 's consisting of second order monomials. Therefore, it can be expressed as a quadratic equation $p Q p^{t}=0$ for an appropriately determined $n \times n$ symmetric matrix $Q$.

The simplex $\Delta(S)$ is identified with the projection on the first $(n-1)$ coordinates, $\tilde{\Delta}(\mathbf{S})=\left\{p \in \mathbb{R}_{+}^{n-1}: \sum_{i=1}^{n-1} p_{i} \leq 1\right\}$. For convenience, $I_{a b}$ also denotes the projected image on $\tilde{\Delta}(\mathbf{S})$. Then, substituting $p_{n}$ with $\left(1-p_{1}-\cdots-p_{n-1}\right)$, we have the following expression for $I_{a b}$ in $\tilde{\Delta}(\mathbf{S})$ :

$$
I_{a b}=\left\{p \in \tilde{\Delta}(\mathbf{S}): p \tilde{Q} p^{t}+p \tilde{q}+c=0\right\}
$$

for some $(n-1) \times(n-1)$ symmetric matrix $\tilde{Q}$, some $(n-1)$-vector $\tilde{q}$ and some real number c.

Expected utilities are a special class of weighted utilities where $\alpha_{k}=\alpha_{k^{\prime}}$ for all $k, k^{\prime} \in \mathbf{Z}$. Then, the equation in (3.c) becomes $\sum_{i=1}^{n} u_{a_{i}} p_{i}=\sum_{i=1}^{n} u_{b_{i}} p_{i}$, which determines an affine subspace of $\Delta(\mathrm{S})$. Moreover, since an affine surface is a special form of a quadratic surface, a weighted utility which is not an expected utility may generate a border which is affine. Example 3.2 illustrates this possibility - since only two acts are observed, it illustrates that a weighted utility may generate an EU-rational behavioral partition.

Example 3.2 : Let $\mathrm{S}$ and $\mathrm{Z}$ be as in Example 2.3 and let acts $a$ and $b$ be as follows;

$$
a:\left\{\begin{array}{l}
s_{1} \rightarrow z_{3} \\
s_{2} \rightarrow z_{2} \\
s_{3} \rightarrow z_{1}
\end{array} \quad b:\left\{\begin{array}{l}
s_{1} \rightarrow z_{3} \\
s_{2} \rightarrow z_{1} \\
s_{3} \rightarrow z_{2}
\end{array}\right.\right.
$$

Consider a preference $\succeq$ represented by a weighted utlity such that $z_{3} \succ z_{2} \succ z_{1}$ and indifference surfaces are as shown in Figure $3(\mathrm{~b})$. For $p \in \Delta(\mathbf{S})$ such that $p_{2}=p_{3}$, we have $a(p)=b(p)$ so that $p \in I_{a b}$. Consider $p$ such that $p_{2}>p_{3}$. Define $\hat{p}$ from $p$ as $\hat{p}_{1}=p_{1}$ and $\hat{p}_{2}=\hat{p}_{3}$. Then, $a(p)$ is obtained by moving $a(\hat{p})$ toward $z_{2}$ parallel to the line $\overline{z_{1} z_{2}}$. From the shape of indifference surfaces, $a(p) \succ a(\hat{p})$. On the other hand, by similar argument, $b(\hat{p}) \succ b(p)$. Since $a(\hat{p}) \sim b(\hat{p})$, we conclude $a(p) \succ b(p)$ if $p_{2}>p_{3}$. Analogously, $b(p) \succ a(p)$ if $p_{2}<p_{3}$. Therefore, $I_{a b}=\left\{p \in \Delta(\mathbf{S}): p_{2}=p_{3}\right\}$ so that the behavioral partition is EU-rational. 


\section{Figure 3}
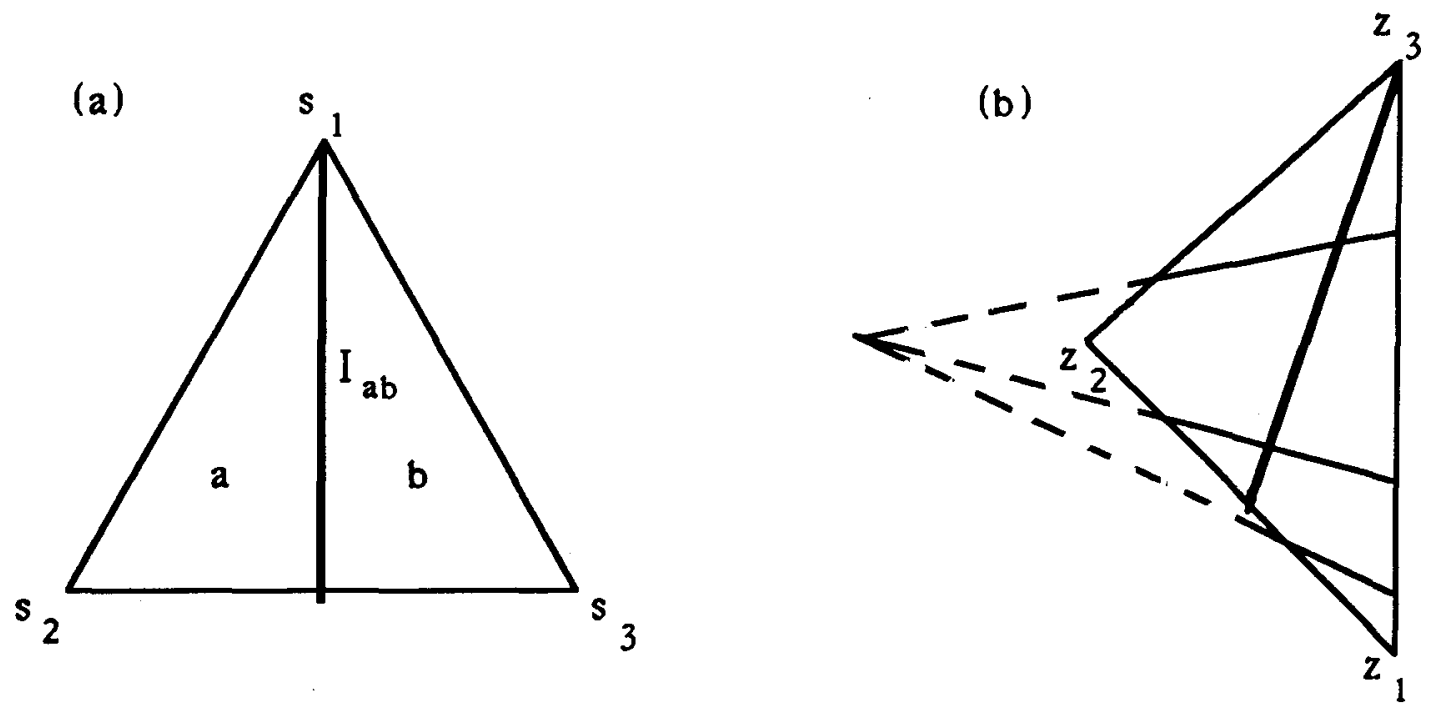

\section{Non-Distinction of Revealed Preferences for "Symmetric" Acts}

Example 3.2 shows that an EU-rational partition can be generated from a weighted utility which is not an expected utility. Nonetheless, if those weighted utilities that may produce an EU-rational behavioral partition are "negligible," given an EU-rational partition, we may still "safely" (in the sense of probability 1) reject the hypothesis that it is produced from a weighted utility. In this respect, we ask how large the set of weighted utilities that may produce EU-rational partitions is.

The answer depends on acts. A pair of acts always admits an affine border under a weighted utility if $i$ ) one of the acts is constant, that is, it leads to the same consequence regardless of states, or $i i$ ) they are "symmetric" in the sense specified below. In this section, pairs of acts of these kinds are investigated. For other pairs of acts, the weighted utilities that may produce affine borders are contained in a "negligible" set in the space of weighted utilities. These pairs of acts are examined in the next section.

Lemma 4.1 : Let $a$ and $b$ acts which are functions from a finite set $\mathbf{S}$ of states to a finite set $\mathrm{Z}$ of consequences. Suppose $a$ is constant. Then, the border $I_{a b}$ is affine under a weighted utility defined over random prospects of $\mathbf{Z}$. 
Proof : Let $z$ be the consequences that $a$ leads to and let $\mathcal{I}_{z} \subset \Delta(Z)$ denote the indifference surface of the sure consequence $z$. Then, $\mathcal{I}_{z}$ is affine. Suppose $p$ and $p^{\prime}$ are in $I_{a b}$. Then, $b(p)$ and $b\left(p^{\prime}\right)$ are in $\mathcal{I}_{z}$. Let $q$ be a convex combination of $p$ and $p^{\prime}$ and consider $b(q)$. Since $b$ is affine as a function from $\Delta(\mathbf{S})$ to $\Delta(Z), b(q)$ is a convex combination of $b(p)$ and $b\left(p^{\prime}\right)$. Since $\mathcal{I}_{z}$ is affine, $b(q)$ belongs to $\mathcal{I}_{z}$, and therefore, $q$ belongs to $I_{a b}$.

Definition 4.2 : Let $\mathrm{S}$ and $\mathrm{Z}$ be finite sets of states and consequences, respectively. Let $a$ and $b$ are acts. Acts $a$ and $b$ are weakly symmetric if $S$ is the union of disjoint subsets $\mathbf{S}^{1}$ and $\mathbf{S}^{2}$ such that each act is constant in $\mathbf{S}^{1}$ and in $\mathbf{S}^{2}$ separately, and the number of consequences induced by $a$ and $b$ is at most three. Acts $a$ and $b$ are strongly symmetric if $\mathbf{S}$ is the union of disjoint subsets $\mathbf{S}^{1}, \mathbf{S}^{2}$ and $\mathbf{S}^{3}$ such that each act is constant in each $\mathbf{S}^{i}(i=1,2,3)$, the consequence that a ( $b$, respectively) leads to in $\mathbf{S}^{1}$ is the consequence that $b$ ( $a$, respectively) leads to in $\mathbf{S}^{2}$, and both acts lead to the same consequence in $\mathbf{S}^{3}$. $A$ pair of acts are symmetric if they are either weakly symmetric or strongly symmetric .

Lemma 4.3 : Given a finite set $\mathrm{S}$ of states and a finite set $\mathrm{Z}$ of consequences, let $a$ and $b$ are symmetric acts. Then, the border $I_{a b}$ is affine under a preference $\succeq$ on $\Delta(Z)$ which is represented by a weighted utility.

Proof : Suppose $a$ and $b$ are weakly symmetric and let $\mathbf{S}^{1}$ and $\mathbf{S}^{2}$ be as in Definition 4.2. Let $a_{i}$ be the consequence of $a$ in $\mathbf{S}^{i}, i=1,2$, and similarly for $b_{i}$. If $a_{i} \succeq b_{i}, i=1,2$, then $I_{a b}$ is one of the followings depending on which preference relation is strict: $\emptyset, \Delta\left(\mathbf{S}^{1}\right)$, $\Delta\left(\mathbf{S}^{2}\right)$ or $\Delta(\mathbf{S})$. Obviously, these are all affine. If $b_{i} \succeq a_{i}, i=1,2$, then analogous argument applies. The remaining case is $a_{1} \succ b_{1}$ and $b_{2} \succ a_{2}$. (The case $b_{1} \succ a_{1}$ and $a_{2} \succ b_{2}$ is redundant because of the symmetry between acts.) If $a_{1}=a_{2}$ or $b_{1}=b_{2}$, the conclusion follows from Lemma 4.1. If $a_{1}=b_{2}$ or $a_{2}=b_{1}$, as probability moves from $\mathbf{S}^{1}$ to $\mathrm{S}^{2}$, the consequence induced by $a$ improves while that induced by $b$ deteriorates. Hence, by the continuity of $\succeq$, there is a unique number $\theta \in(0,1)$ such that $a(p) \sim b(p)$ if and only if $p\left(\mathbf{S}^{1}\right)=\sum_{s \in \mathbf{S}^{1}} p(s)=\theta$. The set of probability assessments over states that satisfy this equality is obviously affine.

Next, suppose $a$ and $b$ are strongly symmetric and let $\mathbf{S}^{1}, \mathbf{S}^{2}$ and $\mathbf{S}^{3}$ be as in Definition 4.2. Let $a_{i}$ be the consequence of $a$ in $\mathbf{S}^{i}, i=1,2,3$, and similarly for $b_{i}$. Denote $a_{1}=b_{2}$ 
by $z$, and denote $a_{2}=b_{1}$ by $z^{\prime}$. If $z \sim z^{\prime}, I_{a b}=\Delta(\mathbf{S})$. Below, we assume $z \succ z^{\prime}$. Then, $a(p)=b(p)$ if $p\left(\mathbf{S}^{1}\right)=p\left(\mathbf{S}^{2}\right)$ so that $I_{a b}$ contains $\left\{p \in \Delta(\mathbf{S}): p\left(\mathbf{S}^{1}\right)=p\left(\mathbf{S}^{2}\right)\right\}$. Consider $p$ such that $p\left(\mathbf{S}^{1}\right) \neq p\left(\mathbf{S}^{2}\right)$. Without loss of generality, assume $p\left(\mathbf{S}^{1}\right)>p\left(\mathbf{S}^{2}\right)$. Find $\tilde{p}$ such that $\tilde{p}\left(\mathbf{S}^{3}\right)=p\left(\mathbf{S}^{3}\right)$ and $\tilde{p}\left(\mathbf{S}^{1}\right)=\tilde{p}\left(\mathbf{S}^{2}\right)$. Notice $a(\tilde{p}) \sim b(\tilde{p})$. Since $z \succ z^{\prime}$, by Betweenness axiom of the preference, $a(p) \succ a(\tilde{p})$ while $b(\tilde{p}) \succ b(p)$ so that $p \notin I_{a b}$. Therefore, $I_{a b}=\left\{p \in \Delta(\mathbf{S}): p\left(\mathbf{S}^{1}\right)=p\left(\mathbf{S}^{2}\right)\right\}$ which is apparently affine.

As is explained in the proof, to determine the border of a pair of symmetric acts, we can identify states in which both acts are constant and ignore those states for which the two acts lead to the same consequence. In this sense, conceptually there are only two distinct states for a pair of symmetric acts. If there are three or more states for a given pair of acts, that are not identified or ignored in the above sense, the acts are not symmetric.

\section{Distinction of Revealed Preferences for "Antisymmetric" Acts}

We investigate the border of a pair of non-constant acts that are not symmetric. First, suppose there are only two states. Then, the unit simplex of states is a unit interval and the border of a pair of acts typically consists of two or less (possibly no) isolated points. Since an isolated point is trivially affine, the border under a weighted utility is EU-rational if it consists of one point, which happens in a "non-negligible" set of weighted utilities. In the remainder of the section, we consider three or more states.

We define "antisymmetric" pairs of acts more restrictive than "not symmetric" to exclude the case that the number of states reduces down to two after identifying those states in which every act is constant; A pair of acts $\{a, b\}$ are antisymmetric if they are not symmetric and there are at least three states $s, s^{\prime}$ and $s^{\prime \prime}$ such that ordered pairs $(a(s), b(s)),\left(a\left(s^{\prime}\right), b\left(s^{\prime}\right)\right)$ and $\left(a\left(s^{\prime \prime}\right), b\left(s^{\prime \prime}\right)\right)$ are different from one another. A preliminary discussion preceeds the main result of this section.

A semi-algebraic set is a finite union of sets in an open subset of $\mathbb{R}^{N}$ of the form

$$
\left\{x \in \mathbb{R}^{N}: P(x)=0 \text { and } Q_{1}(x)>0 \text { and } \ldots \text { and } Q_{J}(x)>0\right\},
$$

where $P$ and $Q_{1}, \ldots, Q_{J}$ are polynomials in $x_{1}, \ldots, x_{N}$ with real coefficients. It is clear from the definition that the class of semi-algebraic sets is closed under finite unions, finite 
intersections and complementation. $A$ set in an open subset of $\mathbb{R}^{N}$ is negligible if it is a semi-algebraic set whose closure has measure 0 . A set is generic if its complement is negligible.

Given a set of consequences $\mathbf{Z}=\{1, \ldots, K\}$, a weighted utility is a pair of functions $(u, \alpha)$ where $u: \mathbf{Z} \rightarrow \mathbb{R}$ is a utility function for pure consequences and $\alpha: \mathbf{Z} \rightarrow \mathbb{R}++$ is a weighting function. We denote $u=\left(u_{1}, \ldots, u_{K}\right) \in \mathbb{R}^{K}$ and $\alpha=\left(\alpha_{1}, \ldots, \alpha_{K}\right) \in \mathbb{R}_{++}^{K}$. Then, the space of weighted utilities is $\mathbb{R}^{K} \times \mathbb{R}_{++}^{K}$.

The result of this section holds for a generic subset of weighted utilities. To avoid unnecessary technical complication, we start with a generic subset of weighted utilities, namely,

$$
\mathcal{W}=\left\{(u, \alpha) \in \mathbb{R}^{K} \times \mathbb{R}_{++}^{K}: u_{k} \neq u_{j} \text { if } k \neq j\right\}
$$

The same preference may be represented by different weighted utilities. First, we eliminate this redundancy. Suppose $(u, \alpha)$ and $(v, \gamma)$ be two representation of a preference $\succeq$ on $\Delta(\mathbf{Z})$. Without loss of generality, suppose $z_{K} \succ z_{(K-1)} \succ \cdots \succ z_{1}$ where $z_{k}$ denotes the sure event of consequence $k$. Then, for each $k \in\{3, \ldots, K\}$, there is a unique number $\theta$ in the unit interval $(0,1)$ such that $z_{2}$ is indifferent to the lottery of $z_{1}$ with probability $\theta$ and $z_{k}$ with probability $1-\theta$. Since each representation should assign the same weighted utility level to these two lotteries, we have

$$
u_{2}=\frac{\alpha_{1} u_{1} \theta+\alpha_{k} u_{k}(1-\theta)}{\alpha_{1} \theta+\alpha_{k}(1-\theta)} \quad \text { and } \quad v_{2}=\frac{\gamma_{1} v_{1} \theta+\gamma_{k} v_{k}(1-\theta)}{\gamma_{1} \theta+\gamma_{k}(1-\theta)}
$$

Eliminating $\theta$ from $(5 . b)$,

$$
\frac{\alpha_{k}\left(u_{k}-u_{2}\right)}{\alpha_{1}\left(u_{2}-u_{1}\right)}=\frac{\gamma_{k}\left(v_{k}-v_{2}\right)}{\gamma_{1}\left(v_{2}-v_{1}\right)} \quad \forall k=3, \ldots, K
$$

Similarly, there is a unique number $\delta \in(0,1)$ such that the lottery of even probability of $z_{1}$ and $z_{2}$ is indifferent to the lottery of $z_{1}$ with probability $\delta$ and $z_{k}$ with probability $1-\delta$. Again, eliminating $\delta$ from the equalities of the weighted utility levels of these two lotteries by $(u, \alpha)$ and $(v, \gamma)$, we get

$$
\frac{\alpha_{k}\left(u_{k}-u_{1}\right)}{\alpha_{2}\left(u_{2}-u_{1}\right)}=\frac{\gamma_{k}\left(v_{k}-v_{1}\right)}{\gamma_{2}\left(v_{2}-v_{1}\right)} \quad \forall k=3, \ldots, K
$$


Equations (5.c) and (5.d) are necessary for $(u, \alpha)$ and $(v, \gamma)$ to represent the same preference.

Proposition 5.1 : Consider a weighted utility $(u, \alpha) \in \mathcal{W}$. Without loss of generality, suppose $u_{1}<u_{2}<\cdots<u_{K}$. Then, there is a unique weighted utility in

$$
\mathcal{W}^{*} \equiv\left\{(v, \gamma) \in \mathcal{W}: v_{k}=k, k=1,2,3, \text { and } \gamma_{1}=1\right\}
$$

that represent the same preference as $(u, \alpha)$.

Proof : Dividing (5.c) by (5.d) side by side,

$$
\frac{\alpha_{2}\left(u_{k}-u_{2}\right)}{\alpha_{1}\left(u_{k}-u_{1}\right)}=\frac{\gamma_{2}\left(v_{k}-v_{2}\right)}{\gamma_{1}\left(v_{k}-v_{1}\right)} \quad \forall k=3, \ldots, K
$$

Given $(u, \alpha) \in \mathcal{W}$, determine $(v, \gamma) \in \mathcal{W}^{*}$ as follows:

a. Let $v_{k}=k, k=1,2,3$ and $\gamma_{1}=1$.

b. Determine $\gamma_{2}$ and $\gamma_{3}$ from (5.f) and (5.c), respectively, for $k=3$.

c. Determine $v_{4}$ from (5.f) for $k=4$, and then determine $\gamma_{4}$ from (5.c) for $k=4$.

d. Repeat step c inductively for $k=5, \ldots, K$.

This process determines $(v, \gamma)$ uniquely. We need to check that it belongs to $\mathcal{W}^{*}$. Recall $u_{1}<u_{2}<\cdots<u_{K}$. Clearly, $\gamma_{2}>0$ and $\gamma_{3}>0$. Inductively, for $k=4, \ldots, K$, $\left(v_{k}-v_{2}\right)>0$ so that $\gamma_{k}>0$. Therefore, $(v, \gamma) \in \mathcal{W}^{*}$.

It remains to show that $(v, \gamma)$ represents the same preference as $(u, \alpha)$. Consider $\sigma$ and $\mu$ in $\Delta(Z)$. We will show

$$
U_{\sigma} \geq U_{\mu} \Longleftrightarrow V_{\sigma} \geq V_{\mu}
$$

where

$$
U_{\sigma}=\frac{\sum_{k=1}^{K} \alpha_{k} u_{k} \sigma_{k}}{\sum_{k=1}^{K} \alpha_{k} \sigma_{k}} \quad \text { and } \quad V_{\sigma}=\frac{\sum_{k=1}^{K} \gamma_{k} v_{k} \sigma_{k}}{\sum_{k=1}^{K} \gamma_{k} \sigma_{k}}
$$

and similarly for $U_{\mu}$ and $V_{\mu}$. Notice that, for any random prospect $\sigma^{\prime} \neq z_{1}, U_{\sigma^{\prime}}>u_{1}$ and $V_{\sigma^{\prime}}>v_{1}$. Hence, if either $\sigma$ or $\mu$ is the sure event $z_{1},(5 . g)$ is immediate.

Next, assume neither $\sigma$ nor $\mu$ is the sure event $z_{1}$ so that all denominators in (5.j) and (5.k) are positive. Notice that (5.c) and (5.d) are satisfied by construction. By substituting 
$\alpha_{k} u_{k}=\frac{A}{B}\left(\gamma_{k} v_{k}-\gamma_{k} v_{2}\right)+\alpha_{k} u_{2}$ in the left hand side of $(5 . g)$, where $A=\alpha_{1}\left(u_{2}-u_{1}\right)$ and $B=\gamma_{1}\left(v_{2}-v_{1}\right)$, we get

$$
A\left(V_{\sigma}-v_{2}\right)=B\left(U_{\sigma}-u_{2}\right)\left(\frac{\sum_{k=1}^{K} \alpha_{k} \sigma_{k}}{\sum_{k=1}^{K} \gamma_{k} \sigma_{k}}\right)
$$

By substituting $\alpha_{k} u_{k}=\frac{A^{\prime}}{B^{\prime}}\left(\gamma_{k} v_{k}-\gamma_{k} v_{1}\right)+\alpha_{k} u_{1}$ in the same side of (5.g), where $A^{\prime}=$ $\alpha_{2}\left(u_{2}-u_{1}\right)$ and $B^{\prime}=\gamma_{2}\left(v_{2}-v_{1}\right)$, we get

$$
A^{\prime}\left(V_{\sigma}-v_{1}\right)=B^{\prime}\left(U_{\sigma}-u_{1}\right)\left(\frac{\sum_{k=1}^{K} \alpha_{k} \sigma_{k}}{\sum_{k=1}^{K} \gamma_{k} \sigma_{k}}\right)
$$

Dividing (5.h) by (5.i) side by side, we get

$$
\frac{\alpha_{1} \gamma_{2}}{\alpha_{2} \gamma_{1}}\left(\frac{v_{1}-v_{2}}{V_{\sigma}-v_{1}}+1\right)=\frac{u_{1}-u_{2}}{U_{\sigma}-u_{1}}+1
$$

The same process on the right hand side of $(5 . g)$ produces

$$
\frac{\alpha_{1} \gamma_{2}}{\alpha_{2} \gamma_{1}}\left(\frac{v_{1}-v_{2}}{V_{\mu}-v_{1}}+1\right)=\frac{u_{1}-u_{2}}{U_{\mu}-u_{1}}+1
$$

Since $u_{1}-u_{2}<0$ and $v_{1}-v_{2}<0$, we conclude from (5.j) and (5.k) that $U_{\sigma} \geq U_{\mu}$ if and only if $V_{\sigma} \geq V_{\mu}$. This completes the proof.

By Proposition 5.1, $\mathcal{W}^{*}$ contains all weighted utilities that rank pure consequences as $z_{K} \succ z_{(K-1)} \succ \cdots \succ z_{1}$ and any two weighted utilities in $\mathcal{W}^{*}$ represent different preferences. Hence, the space obtained from $\mathcal{W}$ by eliminating redundancy is the union of as many copies of $\mathcal{W}^{*}$ as the permutation of $\mathbf{Z}$. Since each copy is identical up to permutation, we may consider one representative copy.

Consider the natural mapping $F$ from the subset of $\mathcal{W}$ that rank sure event as above, onto $\mathcal{W}^{*}$. Then, with the utility values of sure events $z_{1}, z_{2}, z_{3}$ and the weight of $z_{1}$ fixed as independent variables, $F$ is a homeomorphism. In fact, it is locally a change of coordinates, which itself is a smooth function of independent variables. So, $F$ is a surjective submersion. Moreover, $F$ maps a semi-algebraic set to a semi-algebraic set. Therefore, the image of a generic subset of $\mathcal{W}$ under $F$ is generic in $\mathcal{W}^{*} .^{(4)}$ Hence, a generic property in

(4) See Park (1993), Lemma 3.3. 
$\mathcal{W}$ is a generic property in $\mathcal{W}^{*}$. In light of this observation, we state a result that holds for generic weighted utilities in $\mathcal{W}$ : behavioral partitions produced by weighted utilities are distinguishable from those produced by expected utilities. The proof is presented at the end of the section.

Proposition 5.2 : Let $\mathbf{S}=\left\{s_{1}, \ldots, s_{n}\right\}$ be states and let $\mathbf{Z}=\{1, \ldots, K\}$ be consequences where $n \geq 3$. Suppose $a$ and $b$ are acts that are antisymmetric and neither is constant. Then, the set of weighted utilities under which the behavioral partition of $\{a, b\}$ is $E U$ rational, is a negligible subset of $\mathcal{W}$.

Proposition 5.2 has an implication on the size of the set of weighted utilities that may produce EU-rational partitions for three or more acts. Corollary 5.3 is the statement whose proof is immediate from Proposition 5.2.

Corollary 5.3 : Suppose $A$ is a finite set of acts such that none of the acts is constant and an act is antisymmetric to any other act in $\mathbf{A}$. Then, the set of weighted utilities that may produce EU-rational partitions of $\mathbf{A}$, is a negligible subset of weighted utilities defined over random prospects of consequences that can be induced from A.

If one of the acts is constant, then the conclusion of Corollary 5.3 is not generally true because a non-affine border may be hidden in the partition cell of the constant act. The following example illustrates this possibility.

Example 5.4 : Let $\mathbf{S}=\left\{s_{1}, s_{2}, s_{3}, s_{4}\right\}$ and $\mathbf{Z}=\left\{z_{1}, z_{2}, z_{3}\right\}$ be the sets of states and consequences and let $\{a, b, c\}$ be the following acts;

$$
a:\left\{\begin{array}{l}
s_{1} \rightarrow z_{3} \\
s_{2} \rightarrow z_{1} \\
s_{3} \rightarrow z_{1} \\
s_{4} \rightarrow z_{1}
\end{array} \quad b:\left\{\begin{array}{l}
s_{1} \rightarrow z_{2} \\
s_{2} \rightarrow z_{2} \\
s_{2} \rightarrow z_{2} \\
s_{4} \rightarrow z_{2}
\end{array} \quad c:\left\{\begin{array}{l}
s_{1} \rightarrow z_{1} \\
s_{2} \rightarrow z_{1} \\
s_{3} \rightarrow z_{2} \\
s_{4} \rightarrow z_{3}
\end{array}\right.\right.\right.
$$

Notice that neither $\{a, c\}$ nor $\{b, c\}$ are symmetric. First, consider the expected utility according to which $z_{3} \succ z_{2} \succ \dot{z}_{1}$, and $z_{2}$ is indifferent to the lottery of $z_{1}$ with probability $2 / 3$ and $z_{3}$ with probability $1 / 3$. Using the diagram in Figure 4, $a$ is chosen in the top tetrahedron, $c$ is chosen in the bottom tetrahedron and $b$ is chosen in between. $I_{a c}$ is the shaded triangle. Notice that $I_{a c}$ is entirely contained in the interior of the cell for 
b. The indifference surfaces change continuously as the preference changes continuously in the space of weighted utilities. Hence, in the space of weighted utilities, there is a neighborhood of the expected utility that we considered above such that, if we pick a preference in that neighborhood then the corresponding $I_{a c}$ (which is not affine in general) is hidden in the partition cell for $b$. So, those preferences in that neighborhood produce EU-rational partitions.

We close the section by proving Proposition 5.2. A mathematical result to be used in the proof is presented first.

Lemma 5.5 : The solution set to a non-vacuous polynomial in $\mathbb{R}^{N}$ is a negligible subset of $\mathbb{R}^{N}$.

Proof : This is immediate as a special case of Theorem 1 and Theorem 2 of Whitney (1957).

\section{Figure 4}

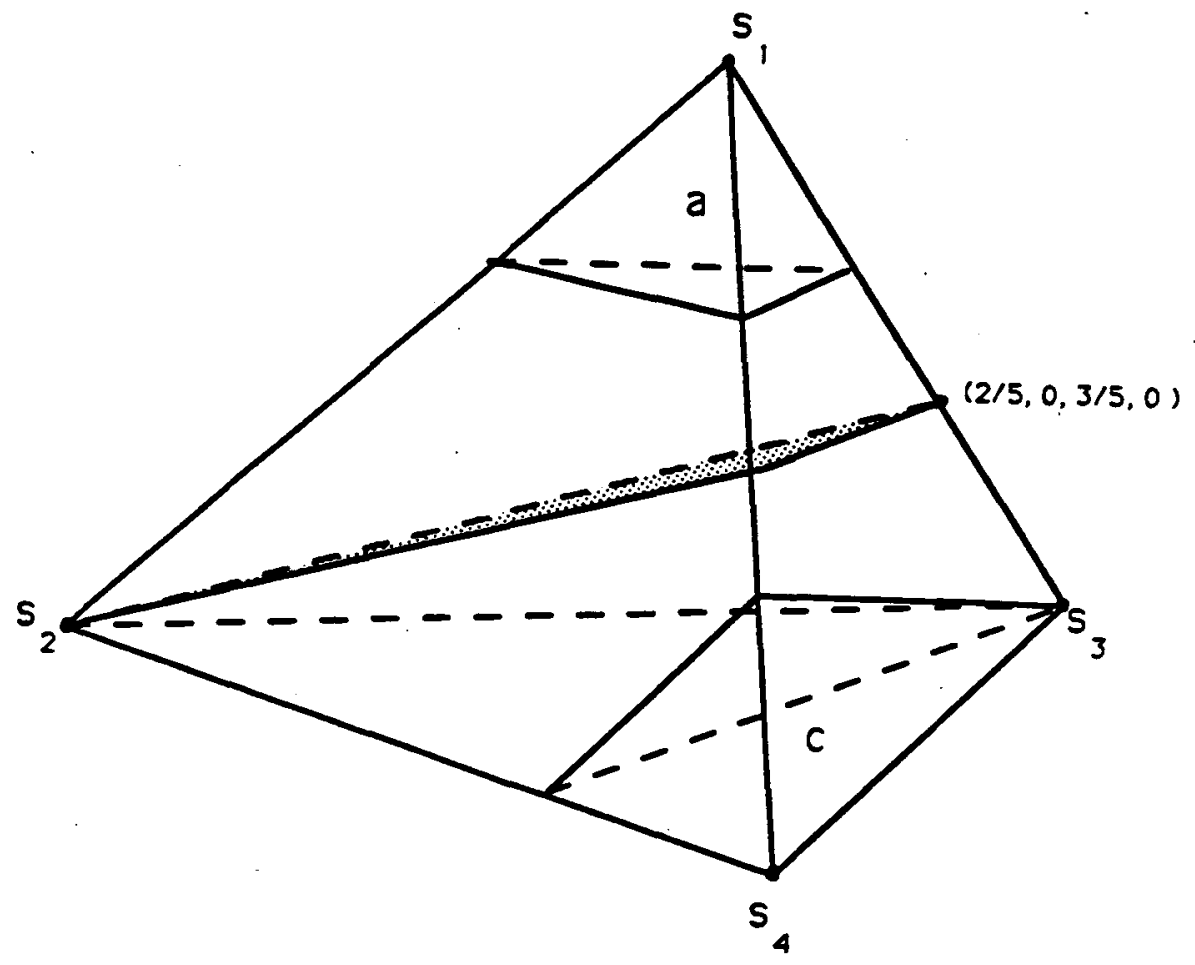


Proof of Proposition 5.2 : It suffices to show the result for the subset of $\mathcal{W}$ that rank pure consequences as $z_{K} \succ z_{(K-1)} \succ \cdots \succ z_{1}$. Hence, we assume in the rest of the proof that the consequence denoted by a higher integer is preferred to one denoted by a lower integer.

Let $a_{i} \in Z$ denotes the consequence induced by $a$ in state $s_{i}$, and similarly for $b_{i}$. Suppose $(u, \alpha)$ is a weighted utility that ranks pure consequences as above and induces an EU-rational behavioral partition of $\{a, b\}: I_{a b}$ is an affine subspace of $\Delta(\mathbf{S})$ that divides $\Delta(\mathbf{S})$ into two parts with nonempty interior. Then, $a_{i}>b_{i}$ for at least one $s_{i}$ and $b_{j}>a_{j}$ for at least one $s_{j}$. Without loss of generality, assume

$$
a_{n}>b_{n} \text { and } b_{k}>a_{k}
$$

Since $a$ and $b$ are antisymmetric, there is another state $s_{h}$ such that $\left(a_{h}, b_{h}\right)$ is different from $\left(a_{n}, b_{n}\right)$ and $\left(a_{k}, b_{k}\right)$. Define $\tilde{\Delta}\left(s_{h}, s_{k}\right)=\left\{\left(p_{h}, p_{k}\right) \in \mathbb{R}_{+}^{2}: p_{h}+p_{k} \leq 1\right\}$ as naturally embedded in $\tilde{\Delta}(\mathbf{S})$. Notice that $a$ is preferred to $b$ at $(0,0) \in \tilde{\Delta}\left(s_{h}, s_{k}\right)$ and the preference is reversed at $(0,1) \in \tilde{\Delta}\left(s_{h}, s_{k}\right)$. So, $I_{a b} \cap \tilde{\Delta}\left(s_{h}, s_{k}\right)$ ia an affine subspace of $\tilde{\Delta}\left(s_{h}, s_{k}\right)$ with codimension 1. From (3.d), $I_{a b} \cap \tilde{\Delta}\left(s_{h}, s_{k}\right)$ is the solution set to the polynomial equation

$$
P\left(p_{h}, p_{k}\right) \equiv \gamma_{h h} p_{h}^{2}+\gamma_{k k} p_{k}^{2}+\gamma_{h k} p_{h} p_{k}+\delta_{h} p_{h}+\delta_{k} p_{k}+c=0
$$

Here the coefficients are as follows $(j=h, k)$ :

$$
\begin{aligned}
\gamma_{j j}= & \left(\alpha_{a_{j}} u_{a_{j}}-\alpha_{a_{n}} u_{a_{n}}\right)\left(\alpha_{b_{j}}-\alpha_{b_{n}}\right) \\
& -\left(\alpha_{b_{j}} u_{b_{j}}-\alpha_{b_{n}} u_{b_{n}}\right)\left(\alpha_{a_{j}}-\alpha_{a_{n}}\right), \\
\gamma_{h k}= & \left(\alpha_{a_{h}} u_{a_{h}}-\alpha_{a_{n}} u_{a_{n}}\right)\left(\alpha_{b_{k}}-\alpha_{b_{n}}\right) \\
& -\left(\alpha_{b_{k}} u_{b_{h}}-\alpha_{b_{n}} u_{b_{n}}\right)\left(\alpha_{a_{k}}-\alpha_{a_{n}}\right) \\
& +\left(\alpha_{a_{k}} u_{a_{k}}-\alpha_{a_{n}} u_{a_{n}}\right)\left(\alpha_{b_{h}}-\alpha_{b_{n}}\right) \\
& -\left(\alpha_{b_{k}} u_{b_{k}}-\alpha_{b_{n}} u_{b_{n}}\right)\left(\alpha_{a_{n}}-\alpha_{a_{n}}\right) \\
\delta_{j}= & \left(\alpha_{a_{j}} u_{a_{j}}-\alpha_{a_{n}} u_{a_{n}}\right) \alpha_{b_{n}}+\alpha_{a_{n}} u_{a_{n}}\left(\alpha_{b_{j}}-\alpha_{b_{n}}\right) \\
& -\left(\alpha_{b_{j}} u_{b_{j}}-\alpha_{b_{n}} u_{b_{n}}\right) \alpha_{a_{n}}-\alpha_{b_{n}} u_{b_{n}}\left(\alpha_{a_{j}}-\alpha_{a_{n}}\right), \\
c= & \alpha_{a_{n}} \alpha_{b_{n}}\left(u_{a_{n}}-u_{b_{n}}\right) \neq 0
\end{aligned}
$$


We check necessary conditions for the solution set to $(5 . m)$ to be affine:

If $\gamma_{h h}=\gamma_{k k}=0$, necessary conditions are

$$
\begin{aligned}
& \text { (c.1) } \gamma_{h k}=0 \text { or } \\
& \text { (c.2) } \gamma_{h k} c=\delta_{h} \delta_{k} .
\end{aligned}
$$

If $\gamma_{h h} \neq 0, P\left(p_{h}, p_{k}\right)=0$ implies

$$
p_{h}=\frac{-\left(\gamma_{h k} p_{k}+\delta_{h}\right) \pm\left[\left(\gamma_{h k} p_{k}+\delta_{h}\right)^{2}-4 \gamma_{h h}\left(\gamma_{k k} p_{k}^{2}+\delta_{k} p_{k}+c\right)\right]^{\frac{1}{2}}}{2 \gamma_{h h}}
$$

This equation is affine in $p_{k}$ only if the formula that we took the square root of is expressed as a square of another formula, that is,

$$
\left(\delta_{h}^{2}-4 \gamma_{h h} c\right)\left(\gamma_{h k}-4 \gamma_{h h} \gamma_{k k}\right)-\left(\gamma_{h k} \delta_{h}-2 \gamma_{h h} \delta_{k}\right)^{2}=0
$$

Finally, if $\gamma_{k k} \neq 0$, analogous argument leads to the necessary condition

$$
\left(\delta_{k}^{2}-4 \gamma_{k k} c\right)\left(\gamma_{h k}-4 \gamma_{h h} \gamma_{k k}\right)-\left(\gamma_{h k} \delta_{k}-2 \gamma_{k k} \delta_{h}\right)^{2}=0
$$

What we will show is that given a pair of acts $a$ and $b$ as specified in Proposition 5.2 , those weighted utilities $(u, \alpha)$ that satisfy one of the necessary conditions (c.1) to (c.4) form a negligible set. By virtue of Lemma 5.5, we only need to show that each condition is a non-vacuous polynomial in $(u, \alpha)$. The analysis depends on the consequences that each act induces. If every consequence from each act in each state is distinct from one another, obviously all conditions are non-vacuous polynomials and the conclusion follows. If some consequences are identical, the analysis involves simple but laborious calculation. For each possible cases, we find a monomial which does not vanish. The result is summarized below.

Since acts are not symmetric, we can choose $s_{n}$ and $s_{k}$ such that $\left(a_{n}, b_{n}\right) \neq\left(b_{k}, a_{k}\right)$. For notational convenience, denote $a_{n}=s$ and $b_{n}=r$ where $s>r$.

Case 1. $a_{k}=s<b_{k}=t$.

In this case $\gamma_{k k}$ is a vacuous polynomial (i.e, identically 0 ) and there is $s_{h}$ such that $a_{h} \neq s$ because $a$ is not constant.

[Subcase 1-1] $\gamma_{h h}=0$.

From the expression of $\gamma_{h h}$ in terms of $(u, \alpha)$, this subcase happens only if either $a_{h}=r$ and $b_{h}=s$, or $b_{h}=b_{n}=r$. 
In this subcase, condition (c.1) is

$$
\left(\alpha_{a_{h}} u_{a_{h}}-\alpha_{s} u_{s}\right)\left(\alpha_{t}-\alpha_{r}\right)-\left(\alpha_{t} u_{t}-\alpha_{r} u_{r}\right)\left(\alpha_{a_{h}}-\alpha_{s}\right)=0
$$

So, the term $\alpha_{s} \alpha_{t} u_{s}$ does not vanish.

Next, condition (c.2) expressed in terms of $(u, \alpha)$ is

$$
\begin{aligned}
& \quad\left[\left(\alpha_{a_{h}} u_{a_{h}}-\alpha_{s} u_{s}\right)\left(\alpha_{t}-\alpha_{r}\right)-\left(\alpha_{t} u_{t}-\alpha_{r} u_{r}\right)\left(\alpha_{a_{h}}-\alpha_{s}\right)\right] \cdot\left[\alpha_{s} \alpha_{r}\left(u_{s}-u_{r}\right)\right] \\
& -\left[\left(\alpha_{a_{h}} u_{a_{h}}-\alpha_{s} u_{s}\right) \alpha_{r}+\alpha_{s} u_{s}\left(\alpha_{b_{h}}-\alpha_{r}\right)-\left(\alpha_{b_{h}} u_{b_{h}}-\alpha_{r} u_{r}\right) \alpha_{s}+\alpha_{r} u_{r}\left(\alpha_{a_{h}}-\alpha_{s}\right)\right] . \\
& \quad\left[\alpha_{s} u_{s}\left(\alpha_{t}-\alpha_{r}\right)-\left(\alpha_{t} u_{t}-\alpha_{r} u_{r}\right) \alpha_{s}\right] \\
& =0
\end{aligned}
$$

Consider the first possibility of $\gamma_{h h}=0: a_{h}=r$ and $b_{h}=s$. Then, the term with $u_{s}^{2}$ is $\alpha_{s}^{2} \alpha_{r}\left(\alpha_{t}-\alpha_{r}\right) u_{s}^{2}$ which does not vanish. Consider the second possibility: $b_{h}=b_{n}=r$. If $a_{h}=t$, the term $\alpha_{t}^{2} \alpha_{r} \alpha_{s} u_{t}^{2}$ does not vanish. If $a_{h} \neq t$, the term $\alpha_{t} \alpha_{s} \alpha_{r} \alpha_{a_{h}} u_{t} u_{s}$ does not vanish.

[Subcase 1-2] $\gamma_{h h} \neq 0$.

Since $\gamma_{k k}=0$, condition (c.3) is $\gamma_{h h}\left(\gamma_{h k} \delta_{h} \delta_{k}-\gamma_{h k} \delta_{k}^{2}-\gamma_{h k}^{2} c\right)=0$. Being too long, the expression in terms of $(u, \alpha)$ is omitted. Similar investigation to Subcase 1-1 is carried out and results are summarized below.

1) If $b_{h}=s$, the term $\alpha_{s}^{3} \alpha_{t}^{2} \alpha_{a_{h}} u_{s}^{3}$ does not vanish.

2) If $b_{h}=t=a_{h}$, the term $\alpha_{t}^{2} \alpha_{s}^{2} \alpha_{r}^{2} u_{t}^{2} u_{s}$ does not vanish.

3) If $b_{h}=t$ but $a_{h} \neq t$, the term $\alpha_{t}^{3} \alpha_{s}^{3} u_{t}^{2} u_{s}$ does not vanish.

4) If $b_{h} \neq s, b_{h} \neq t$ and $a_{h}=t$, the term $\alpha_{t}^{2} \alpha_{s}^{2} \alpha_{r}^{2} u_{t}^{2} u_{s}$ does not vanish.

5) If $b_{h} \neq s, b_{h} \neq t$ and $a_{h} \neq t$, the term $\alpha_{t}^{2} \alpha_{s}^{2} \alpha_{a_{h}} \alpha_{b_{h}} u_{t}^{2} u_{s}$ does not vanish.

Case 2. $a_{k}=d<b_{k}=r$.

By exchanging the roles of $a$ and $b$ and those of $s_{n}$ and $s_{k}$, this is the same situation as Case 1.

Case 3. $a_{k}=f$ and $b_{k}=g$ where $f<g, f \neq s, g \neq r$ and $(g, f) \neq(s, r)$.

Denote $a_{h}=x$ and $b_{h}=y$ and recall that $(x, y) \neq(s, r)$ and $(x, y) \neq(f, g)$. From these conditions, it is easily checked that $\gamma_{k k}$ is not a vacuous polynomial. So, we consider 
condition (c.4). Again, we omit the detailed process and summarize results for various cases: we divide cases depending on whether $g, x$ and $y$ are equal to $s$ or not, and in each case we consider further variation.

1) $g=s, x=s, y=s$ : Then, $f \neq r$ because $(g, f) \neq(s, r)$. In this case, the term $\alpha_{s}^{4} \alpha_{r}^{3} \alpha_{f} u_{s}^{4}$ does not vanish.

2) $g=s, x=s, y \neq s$ : Then, $f \neq r$ because $(g, f) \neq(s, r)$ and $y \neq r$ because $(x, y) \neq(s, r)$. In this case, the term $\alpha_{s}^{4} \alpha_{r}^{3} \alpha_{y} u_{s}^{4}$ does not vanish.

3) $g=s, x \neq s, y=s$ : Then, $f \neq r$ because $(g, f) \neq(s, r)$ and $f \neq x$ because $(x, y) \neq(f, g)$. In this case, the term $\alpha_{s}^{4} \alpha_{f}^{3} \alpha_{r} u_{s}^{4}$ does not vanish.

4) $g \neq s, x=s, y=s$ : Recall $f \neq g$ and $g \neq r$. In this case, the term $\alpha_{s}^{4} \alpha_{g}^{2} \alpha_{r} \alpha_{f} u_{s}^{4}$ does not vanish.

5) $g=s, x \neq s, y \neq s$ : Then, $f \neq r$ because $(g, f) \neq(s, r)$.

If $x=f$ and $y \neq f$, the term $\alpha_{s}^{4} \alpha_{f}^{3} \alpha_{r} u_{s}^{4}$ does not vanish.

In all other situations, the term $\alpha_{s}^{4} \alpha_{f}^{4} u_{s}^{4}$ does not vanish.

6) $g \neq s, x=s, y \neq s$ : Then, $y \neq r$ because $(x, y) \neq(s, r)$.

If $y=g$, the term $\alpha_{s}^{3} \alpha_{g}^{3} \alpha_{f} \alpha_{r} u_{s}^{3} u_{g}$ does not vanish.

If $y \neq g$, the term $\alpha_{s}^{4} \alpha_{g}^{2} \alpha_{y}^{2} u_{s}^{3} u_{g}$ does not vanish.

7) $g \neq s, x \neq s, y=s$ : Recall $f \neq g$ and $g \neq r$.

If $x=g$, the term $\alpha_{s}^{4} \alpha_{g}^{4} u_{s}^{4}$ does not vanish.

If $x \neq g$, the term $\alpha_{s}^{4} \alpha_{g}^{3} \alpha_{x} u_{s}^{4}$ does not vanish.

8) $g \neq s, x \neq s, y \neq s$ :

If $x=y=g$, the term $\alpha_{s}^{3} \alpha_{g}^{3} \alpha_{r}^{2} u_{s}^{3} u_{g}$ does not vanish.

If $x=g$ but $y \neq g$, the term $\alpha_{s}^{3} \alpha_{g}^{4} \alpha_{y} u_{s}^{3} u_{g}$ does not vanish.

If $x \neq g$ but $y=g$, the term $\alpha_{s}^{3} \alpha_{g}^{4} \alpha_{x} u_{s}^{3} u_{g}$ does not vanish.

If $x \neq g$ and $y \neq g$, the term $\alpha_{s}^{3} \alpha_{g}^{3} \alpha_{x} \alpha_{y} u_{s}^{3} u_{g}$ does not vanish.

Until now, we showed that, given an antisymmetric pair of non-constant acts $a$ and $b$, those weighted utilities $(u, \alpha)$ that satisfy one of the conditions (c.1) to (c.4) form a negligible set. Since one of these conditions is necessary for a behavioral partition to be EU-rational, the proof of Proposition 5.2 is complete. 


\section{Concluding Remarks}

Economic theories may not describe the precise picture of the real world, but need to give a good scientific explanation about the observed economic behavior. In this sense, given an EU-rational observation, expected utility theory can be regarded as appropriate even if the observation might not have resulted from expected-utility maximizing behavior. However, when one makes predictions based on EU-rational observations, one needs to be more careful: properties inconsistent with expected utility may be dormant and become revealed when a new feasible act is added. Proposition 5.2 and Corollary 5.3 provide one criterion to exclude this possibility with probability 1 .

In adopting expected utility or alternative theories as the relevant behavioral framework of agents in a given situation involving uncertainty, empirical evidence plays an important role if available. For this reason, we need a knowledge of revealed behavior patterns of alternative theories as well as that of expected utility. This paper presents a necessary condition of the revealed preference of weighted utility. For a complete characterization of weighted utility, a sufficient condition is needed analogous to the integrability condition of Green and Osband. Moreover, studies on other non-expected utility theories may be useful for a more comprehensive understanding of the behavior under uncertainty. 


\section{REFERENCES}

Border, K. (1992), "Revealed Preference, Stochastic Dominance and the Expected Utility Hypothesis," Journal of Economic Theory, 56, 20-42.

Chew, S. H. (1983), "A Generalization of the Quasilinear Mean with Applications to Income Inequality and Decision Theory Resolving the Allais Paradox," Econometrica, 51, 1065-1092.

Chew, S. H. (1988), “Axiomatic Utility Theories with the Betweenness Property," Annals of Operation Research: Choice under Uncertainty, forthcoming.

Chew, S. H. and L. G. Epstein (1989), "A Unifying Approach to Axiomatic Nonexpected Utility Theories," Journal of Economic Theory, 49, 207-240.

Dekel, E. (1986), "An axiomatic Characterization of Preference under Uncertainty: Weakening the Independence Axiom," Journal of Economic Theory, 40, 304-318.

Fishburn, P. (1970), Utility Theory for Decision Making. Wiley, New York.

Fishburn, P. (1975), "Separation Theorems and Expected Utility," Journal of Economic Theory, 11, 16-34.

Green, E. J. and K. Osband (1991), “A Revealed Preference Theory for Expected Utility,” Review of Economic Studies, 58, 677-696.

Guillemin, V. and A. Pollack (1974), Differential Topology. Prentice-Hall, Inc., New Jersey. Kelley, J. (1955), General Topology. Springer-Verlag.

Kim, T. (1991), "The Subjective Expected Utility Hypothesis and Revealed Preference," Economic Theory, 1, 251-263.

Kreps, D. (1988), Notes on the Theory of Choice. Westview, Boulder.

Machina, M. (1987), "Choice under Uncertainty: Problems Solved and Unsolved," Journal of Economic Perspectives, 1, 121-154.

Machina, M. (1989), "Dynamic Consistency and Non-Expected Utility Models of Choice under Uncertainty," Journal of Economic Literature, 27, 1622-1668.

Park, I.-U. (1993), "Generic Finiteness of Equilibrium Outcome Distributions in SenderReceiver Cheap-Talk Games,' mimeo, Department of Economics, University of Minnesota. 
Quiggin, J. (1982), "Anticipated Utility Theory," Journal of Economic Behavior and Organization, 3, 323-343.

Savage, L. J. (1954), The Foundations of Statistics. Wiley, New York.

von Neumann, J. and O. Morgenstern (1944), Theory of Games and Economic Behavior. Princeton University Press, Princeton.

Whitney, H. (1954), "Elementary Structure of Real Algebraic Varieties," Annals of Mathematics, 66, 545-556.

Yaari, M. E. (1987), "The Dual Theory of Choice under Risk; Risk Aversion without Diminishing Marginal Utility," Econometrica, 55, 95-115. 